\title{
Design and Implementation of a Visual Analytics Electronic Antibiogram within an Electronic Health Record System at a Tertiary Pediatric Hospital
}

Allan F. Simpao ${ }^{1}$ Luis M. Ahumada ${ }^{2}$ Beatriz Larru Martinez ${ }^{3}$ Ana M. Cardenas ${ }^{4}$ Talene A. Metjian ${ }^{5}$ Kaede V. Sullivan ${ }^{6}$ Jorge A. Gálvez ${ }^{1}$ Bimal R. Desai ${ }^{7}$ Mohamed A. Rehman ${ }^{8}$ Jeffrey S. Gerber ${ }^{7}$

\author{
${ }^{1}$ Department of Anesthesiology and Critical Care Medicine, Perelman \\ School of Medicine at the University of Pennsylvania and Children's \\ Hospital of Philadelphia, Philadelphia, Pennsylvania, United States \\ 2 Enterprise Analytics and Reporting, Children's Hospital of \\ Philadelphia, Philadelphia, Pennsylvania, United States \\ ${ }^{3}$ Department of Pediatrics, Children's Hospital Los Angeles, Los \\ Angeles, California, United States \\ ${ }^{4}$ Department of Pathology and Laboratory Medicine, Perelman \\ School of Medicine at the University of Pennsylvania and Children's \\ Hospital of Philadelphia, Philadelphia, Pennsylvania, United States \\ ${ }^{5}$ Antimicrobial Stewardship Program, Children's Hospital of \\ Philadelphia, Philadelphia, Pennsylvania, United States \\ ${ }^{6}$ Department of Pathology and Laboratory Medicine, Lewis Katz \\ School of Medicine at Temple University, Philadelphia, Pennsylvania, \\ United States \\ 7 Department of Pediatrics, Perelman School of Medicine at the \\ University of Pennsylvania and Children's Hospital of Philadelphia, \\ Philadelphia, Pennsylvania, United States \\ ${ }^{8}$ Department of Anesthesiology, Johns Hopkins All Children's \\ Hospital, St. Petersburg, Florida, United States
}

Address for correspondence Allan F. Simpao, MD, MBI, Department of Anesthesiology and Critical Care Medicine, Perelman School of Medicine at the University of Pennsylvania and Children's Hospital of Philadelphia, 3401 Civic Center Boulevard, Philadelphia, PA 191044399, United States (e-mail: simpaoa@email.chop.edu).

Appl Clin Inform 2018;9:37-45.

\section{Abstract}

Keywords

- antibiogram

- electronic health records

- clinical decision support systems

- analytics

- data visualization

- pediatrics

- quality improvement
Background Hospitals use antibiograms to guide optimal empiric antibiotic therapy, reduce inappropriate antibiotic usage, and identify areas requiring intervention by antimicrobial stewardship programs. Creating a hospital antibiogram is a timeconsuming manual process that is typically performed annually.

Objective We aimed to apply visual analytics software to electronic health record (EHR) data to build an automated, electronic antibiogram ("e-antibiogram") that adheres to national guidelines and contains filters for patient characteristics, thereby providing access to detailed, clinically relevant, and up-to-date antibiotic susceptibility data.

Methods We used visual analytics software to develop a secure, EHR-linked, condition- and patient-specific e-antibiogram that supplies susceptibility maps for organisms and antibiotics in a comprehensive report that is updated on a monthly basis. Antimicrobial susceptibility data were grouped into nine clinical scenarios according to the specimen source, hospital unit, and infection type. We implemented the eantibiogram within the EHR system at Children's Hospital of Philadelphia, a tertiary received

August 5, 2017

accepted after revision

November 19, 2017
DOI https://doi.org/ 10.1055/s-0037-1615787. ISSN 1869-0327. 
pediatric hospital and analyzed e-antibiogram access sessions from March 2016 to March 2017.

Results The e-antibiogram was implemented in the EHR with over 6,000 inpatient, 4,500 outpatient, and 3,900 emergency department isolates. The e-antibiogram provides access to rolling 12-month pathogen and susceptibility data that is updated on a monthly basis. E-antibiogram access sessions increased from an average of 261 sessions per month during the first 3 months of the study to 345 sessions per month during the final 3 months.

Conclusion An e-antibiogram that was built and is updated using EHR data and adheres to national guidelines is a feasible replacement for an annual, static, manually compiled antibiogram. Future research will examine the impact of the e-antibiogram on antibiotic prescribing patterns.

\section{Background and Significance}

Hospitals routinely perform antimicrobial susceptibility testing for bacterial pathogens and then summarize the results in a table called an antibiogram. Clinicians refer to antibiograms to guide optimal empiric antibiotic therapy and reduce inappropriate antibiotic usage. ${ }^{1}$ Antibiograms can also be used to track changes in antibiotic resistance over time, perform surveillance for emergence of drug-resistant organisms, and identify areas for intervention by antimicrobial stewardship programs. ${ }^{2,3}$

The Clinical and Laboratory Standards Institute (CLSI) developed consensus guidelines in 2007 (revised in 2014) for the collection, storage, analysis, and presentation of antimicrobial susceptibility data. ${ }^{4,5}$ Hospitals' adherence to these guidelines has varied widely, and a 2013 survey determined that only $39 \%$ of U.S. pediatric hospitals compiled an antibiogram on a yearly basis. ${ }^{6}$ One possible reason for this limited adoption is that creating an antibiogram is typically a labor-intensive, manual process. At our hospital, one physician was responsible for the month-long task of compiling the annual institution-wide antibiogram manually. This hospital-wide antibiogram reported aggregate data with limited granularity within specific patient groups and patient care units. $^{7}$

\section{Objective}

Thus, we embarked on a quality improvement project to automate this manual process by using electronic health record (EHR) data to generate an electronic antibiogram ("e-antibiogram") that adheres to the CLSI guidelines and contains filters for patient age, medical conditions, unit location, and other factors that provide the user with access to more detailed, up-to-date data than a hospital-wide, annually produced antibiogram.

\section{Methods}

This study was performed at the Children's Hospital of Philadelphia (CHOP), an urban, pediatric hospital with
535 beds. The CHOP Institutional Review Board deemed the study protocol exempt from review. The hospital system has approximately 29,000 inpatient admissions yearly and has an ambulatory care network consisting of more than 50 outpatient primary and specialty care locations that receive approximately 1.2 million outpatient visits each year. The hospital's infectious disease diagnostics laboratory (IDDL) processes all microbiology cultures obtained during inpatient, emergency department, and outpatient hospital encounters, and data are recorded in the hospital's EHR (Epic, Verona, Wisconsin, United States) since November 2011. Prior to this study, the IDDL compiled an annual institution-wide antibiogram that aggregated the susceptibility data of isolates that were obtained from all specimen types across the entire hospital.

The IDDL uses the automated VITEK 2 (bioMérieux, Durham, North Carolina, United States) system to perform organism identification and antimicrobial susceptibility testing in conjunction with disk diffusion and gradient strip testing per CLSI guidelines. D-testing for inducible clindamycin resistance was performed for all Staphylococcus aureus isolates. For S. aureus, oxacillin-susceptible isolates were identified as methicillin-susceptible (MSSA), while oxacillin nonsusceptible isolates were identified as methicillin-resistant S. aureus (MRSA). We do not test routinely for extendedspectrum $\beta$-lactamases per CLSI recommendations. Cumulative antimicrobial susceptibilities were calculated separately for MRSA and MSSA isolates. Pathogen antimicrobial susceptibility estimates were based on clinical factors entered by the EHR user and include the following: patient age, specimen type, and anatomic source of culture; location of clinical encounter; and key early features of the microbiologic result, including Gram stain, morphology, and biochemical testing results.

All bacterial isolates originating from positive cultures collected during inpatient and outpatient encounters during the study period were included. In the case of multiple isolates from the same patient, the first isolate of each species from a given patient, in each hospital unit, per year was included. Selective reporting of antibiogram data 
was implemented per CLSI guidelines. Screening and surveillance cultures were excluded. Demographic data in the microbiology records were retrieved and included patient age, sex, and the location where the clinical specimen was obtained (inpatient, outpatient, emergency department). Patient age was classified into one of six groups: newborn (less than 1 month of age), young infant (1-3 months), infant ( 3 months -1 year), child (1-5 years), school child (5-15 years), and adolescent (greater than 15 years). Outpatient locations were grouped by clinical activity into primary care and subspecialty clinics.

\section{Dashboard Development}

As we have described previously, we followed a five-step process to create a secure, EHR-linked, condition- and patient-specific visual analytics e-antibiogram that aggregates and displays bacterial isolate data following the CLSI guidelines. ${ }^{8-10}$ First, we retrieved the relevant data from the EHR relational reporting database (Epic Clarity) and then merged thousands of conventional relational database tables into a single dimensional model within visual analytics software (QlikView, Radnor, Pennsylvania, United States). ${ }^{11}$

-Fig. 1 shows the e-antibiogram's dimensional model diagram based on a snowflake schema (data relationships). The dimensional model presents the data in an intuitive framework that contains information regarding the Procedure Order, Order Result, and Order Sensitivity tables as the "fact" or main table (MAIN_FACT_TABLE). The model also contains the original antibiotic, organism, procedure, and admission, discharge, and transfer tables as well as the curated lookup tables that are described in a later section. This dimensional model is highly denormalized and eliminates the complexity from entity relationship diagrams while providing the same approach to the logical and physical data models. The second step of dashboard development consisted of exploratory data analysis. Histograms and other visual summarizations of the data were created to explore the data structure and perform initial data validation. The third step of the dashboard creation process included data modeling, transformation, and additional validation. The fourth step-information visualization-involved constructing an interactive user interface that adhered to seminal visualization principles to achieve graphical excellence and integrity. ${ }^{12}$ This fourth step included selecting the appropriate colors, eliminating distractors, determining the proper data density, and formulating an aesthetically pleasing "golden rectangle" interface design layout. ${ }^{13}$ Some examples of how these principles were implemented include careful selection of font types and sizes to maximize readability, standardized spacing among and within cells, neutral colors for the areas framing the green-red-or-yellow e-antibiogram cells so as not to distract the clinician, and appropriate shortening of antibiotic and organism names to maintain uniform cell sizes. During the fifth step, iterative usability testing, end-users used the "live" e-antibiogram dashboard and then reported feedback that refined the dashboard's final interface. The evaluators in the fifth step included informatics-trained pediatricians and a usability expert. They provided suggestions on features of the interface including the e-antibiogram's layout and color scheme.

Antimicrobial susceptibility testing results were collapsed into two categories, susceptible and nonsusceptible, and were displayed as a percentage of susceptible isolates. An alert was added when the denominator consisted of fewer than 30 isolates. ${ }^{14}$ Routinely tested antibiotics were included. Infectious diseases specialists at our hospital

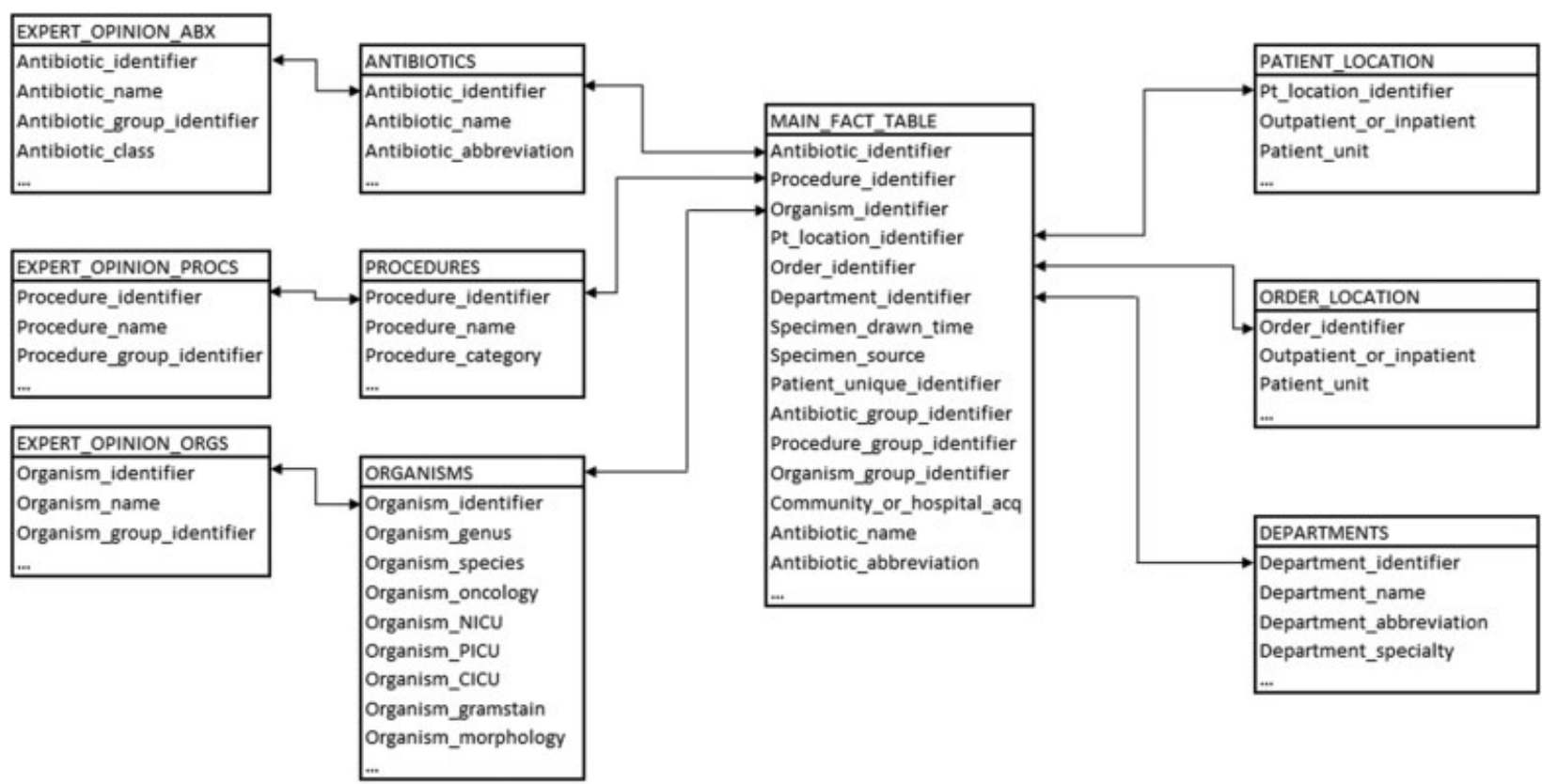

Fig. 1 The electronic antibiogram's dimensional model diagram is based on a snowflake schema. The dimensional model contains information regarding the Procedure Order, Order Result, and Order Sensitivity tables as the "fact" or main table (MAIN_FACT_TABLE). The model also contains the original antibiotic, organism, procedure, and patient and order location tables as well as lookup tables that are curated by infectious disease specialists. 
selected the organisms for inclusion in the antibiogram based on their clinical relevance and the frequency with which they were isolated.

Infectious disease specialists curated the content to five lookup tables (Organisms, Antibiotics, Specimen Source, Procedures, Specimen Taken Location) to map all possible and valid combinations. For example, for the Organisms lookup table, we created groups for Genus, Species, GramNegative/Positive, Morphology, and Special Morphology. These external mappings have enabled us to adapt the e-antibiogram to varying content in different laboratory information systems. While the initial content for our electronic antibiogram came from Meditech (Meditech, Westwood, Massachusetts, United States), the e-antibiogram was adapted easily to SoftLab (SCC Soft Computer, Clearwater, Florida, United States), which has a different set of organism descriptions. The lookup tables are updated monthly based on the frequency of laboratory orders and the dates when they were ordered or processed.

Susceptibility data were grouped into nine clinical scenarios as follows: community-acquired infections (isolates obtained from outpatients, emergency department patients, or inpatients less than 72 hours after hospital admission) sourced from blood, urine, or a wound; hospital-acquired infections (isolates obtained from inpatients more than 72 hours after hospital admission) sourced from blood or urine; and the hospital unit (oncology, pediatric intensive care unit, neonatal intensive care unit, and cardiac intensive care unit).

\section{Dashboard Validation and Clinical Comparisons}

Infectious diseases specialists validated the e-antibiogram using a set of diverse clinical microbiology scenarios, simulating the sequence and timing of information that clinicians receive during their typical clinical workflow. Clinical validation was performed to assess the accuracy and validity of the e-antibiogram. ${ }^{15}$ This validation consisted of the development team exploring a variety of clinically relevant use cases, one of which is included as an example in the "Results" section.

\section{Dashboard Usage}

The e-antibiogram was made accessible to all users via an external link located in a drop-down menu in the EHR. Because the e-antibiogram utilizes Web-accessible visual analytics software, only the operating system login credentials (and not the EHR login credentials) are recorded when the antibiogram is launched. Many EHR workstation terminals at our hospital do not require operating system logins, and the users who access the e-antibiogram on these workstations are recorded as "unknown."

The EHR database was queried for the operating system login credentials of users who launched the e-antibiogram Web link from within the EHR. The users were categorized using their operating system login credentials into the following categories: physician house staff (fellows and residents), attending physicians, pharmacists and nurses, physician assistants and advance practice nurses, and medical and nursing students.

\section{Statistical Analysis}

Descriptive statistics including frequency distributions were performed using Excel (Microsoft Inc., Redmond, Washington, United States). Antimicrobial susceptibilities were compared between cumulative and stratified e-antibiograms using chi-square or Fisher's exact test. Difference among proportions over time was tested using a chi-square test for trend. Exact 95\% confidence intervals for binomial variables were calculated. A two-tailed $p$-value of $<0.05$ was considered statistically significant. All data were analyzed using STATA v.12 software (Stata Inc., College Station, Texas, United States).

\section{Results}

The e-antibiogram was developed, implemented in the EHR, and made available to users in July 2015. More than 6,000 inpatient, 4,500 outpatient, and almost 4,000 emergency department isolates from January 2012 to July 2015 were included in the e-antibiogram. The e-antibiogram is updated monthly based on EHR data. The initial user interface screen consists of the e-antibiogram with nine preselected clinical scenarios. A variety of filtering options frame the central display region and are accessible via open item selectors, drop-down menus, and a date range selector slider.

Once the user has selected a clinical scenario (such as Community-Acquired Infection-Blood), a chart with the frequency distribution of isolated pathogens is displayed (-Fig. 2) followed by a table summarizing the antimicrobial susceptibilities for the antibiotics that are both routinely tested and potentially useful (-Fig. 3). Users also have the option to filter results by either rolling 12-month period or total aggregated data. The e-antibiogram also allows users to explore the pathogen and susceptibility data in granular detail using various tabular and graphical formats (e.g., time series, heat maps).

\section{E-antibiogram Use Case}

To illustrate how an e-antibiogram can aid clinicians to better target empiric antimicrobial therapy, we selected the combination MRSA and clindamycin in the e-antibiogram due to their clinical relevance. MRSA is a leading cause of skin and soft-tissue infections with few oral antibiotic treatment options for children. Significant differences were found between the hospital-wide clindamycin susceptibility of all MRSA isolates and the clindamycin susceptibility showed in unit-specific e-antibiograms. For example, 90\% of wound MRSA isolates obtained in the emergency department were susceptible to clindamycin compared with $79 \%$ in the hospital-wide e-antibiogram [chi-square $(1)=187.74$, $p \leq 0.001]$. Stratifying results by type of specimen (wound) and type of infection (community-acquired) showed significant differences in susceptibility results. During the study period, the clindamycin susceptibility among MRSA isolates was at least consistently $10 \%$ higher among isolates obtained in patients presenting to the emergency department who were discharged home compared with the cumulative 


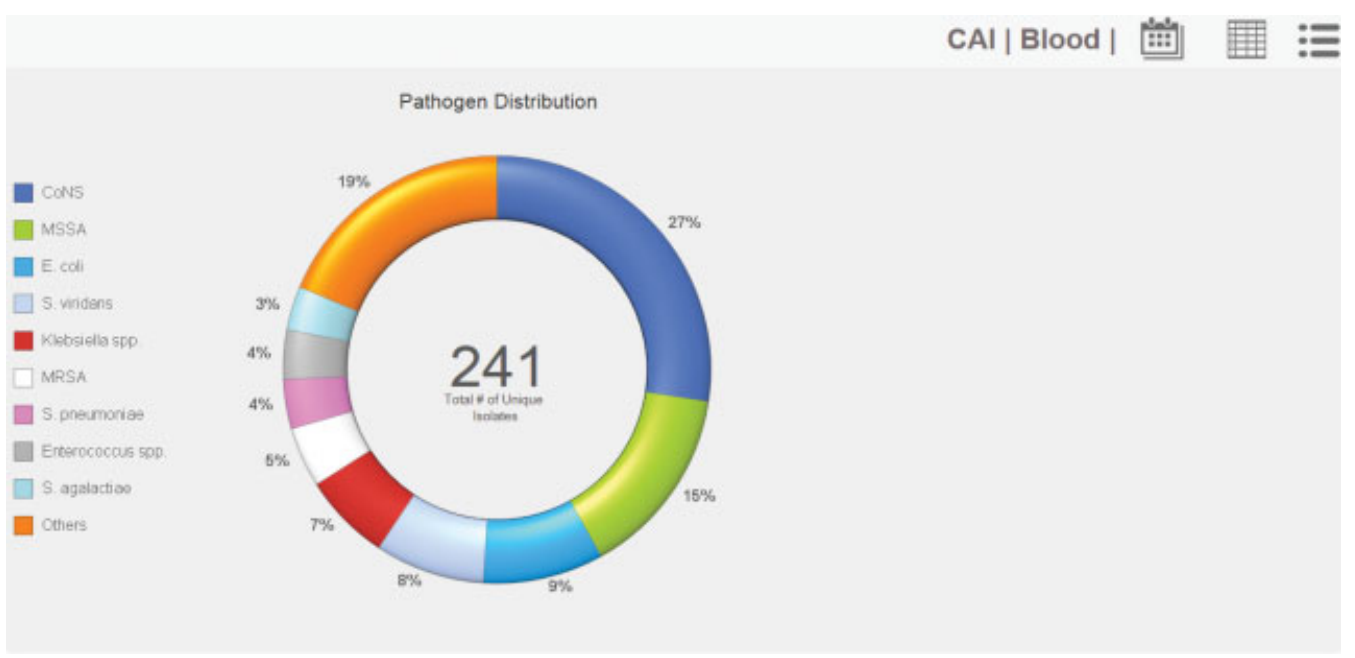

Fig. 2 A screenshot of the user interface for the Community-Acquired Infection (CAI) Blood 12-month case scenario within the electronic antibiogram is shown. The initial selection shows a graph of the distribution of isolated pathogens, while the lower panel shows the antibiotic susceptibility summary. Users can return to the main screen, apply the date filter, and view the pathogens that are contained in the "Others" group. Abbreviations: CoNS, coagulase-negative staphylococci; MRSA, methicillin-resistant Staphylococcus aureus; MSSA, methicillin- susceptible Staphylococcus aureus.

clindamycin susceptibility among all MRSA isolates. The age group filters showed similar results. The demographic data captured in the e-antibiogram showed slightly lower clindamycin susceptibility among wound community-acquired obtained MRSA isolates in neonates (78\%) compared with older children (88\%), although this was not a significant difference between age groups (c.001]. St $=0.37, p=0.71$ ).

\section{E-antibiogram Session Counts}

The e-antibiogram sessions counts have increased steadily since the tool's release. Monthly usage data during the study period is shown in -Fig. 4 and the distribution of usage across the various user categories is listed in - Table 1.

An average of 11 e-antibiogram sessions per day were recorded over a 12-month period across both antibiotic prescribers (attending physicians, fellows, residents, and interns) and nonprescribers (pharmacists, nurses). E-antibiogram session counts increased from an average of 261 sessions per month during the first 3 months of the study to 345 sessions per month during the final 3 months. During the study period, on workstations that required login credentials, physicians, pharmacists, and nurses accounted for the majority of e-antibiogram session totals.

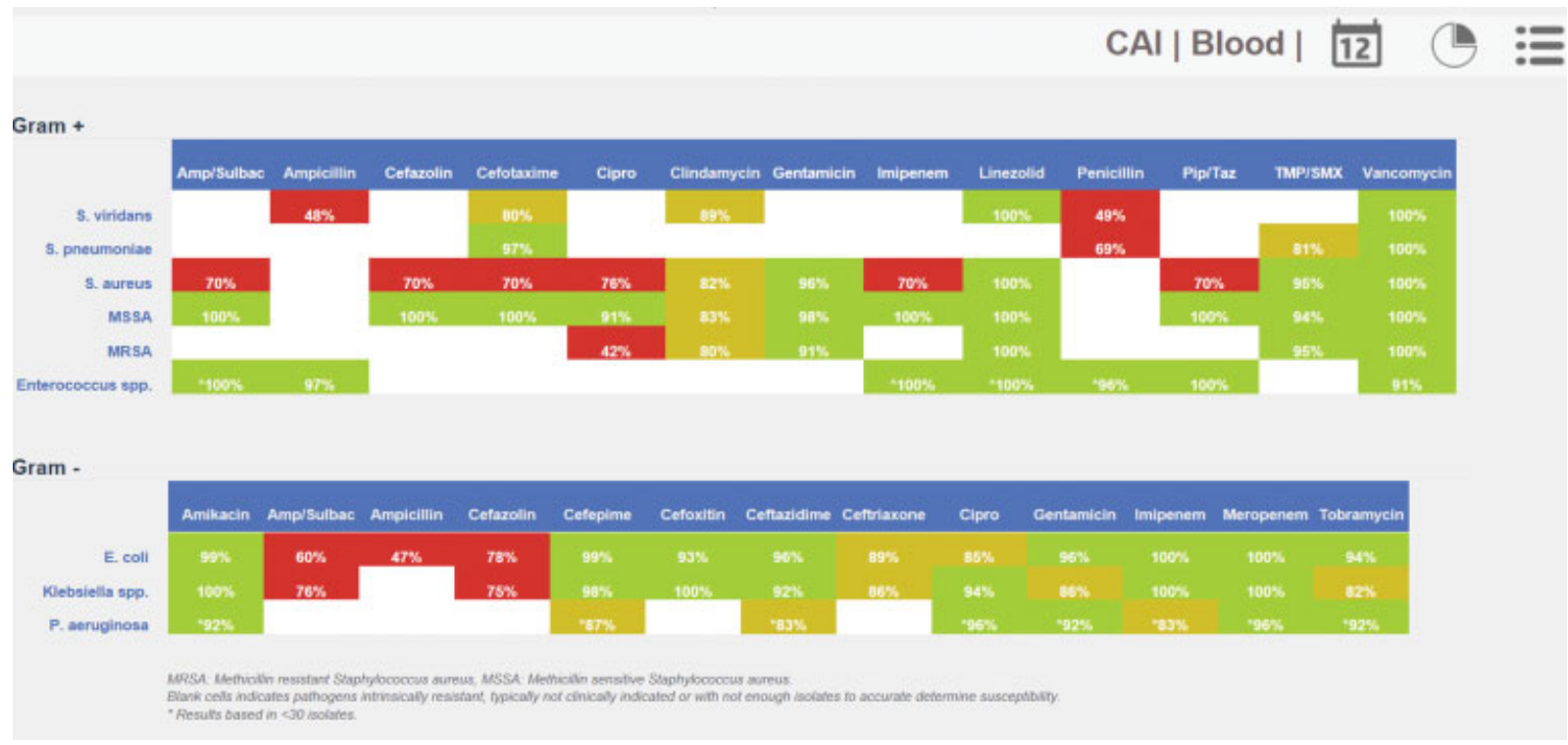

Fig. 3 A screenshot is shown of the user interface for the Community-Acquired Infection (CAI) Blood case scenario antibiotic susceptibility summary within the electronic antibiogram. Users can use the icons in the top right to return to the main screen, apply the date filter, and return to the summary graph. Abbreviations: Amp/Sulbac, ampicillin-sulbactam; Cipro, ciprofloxacin; MRSA, methicillin-resistant Staphylococcus aureus; MSSA, methicillin-susceptible Staphylococcus aureus; Pip/Taz, piperacillin/tazobactam; TMP/SMX, trimethoprim/sulfamethoxazole. 


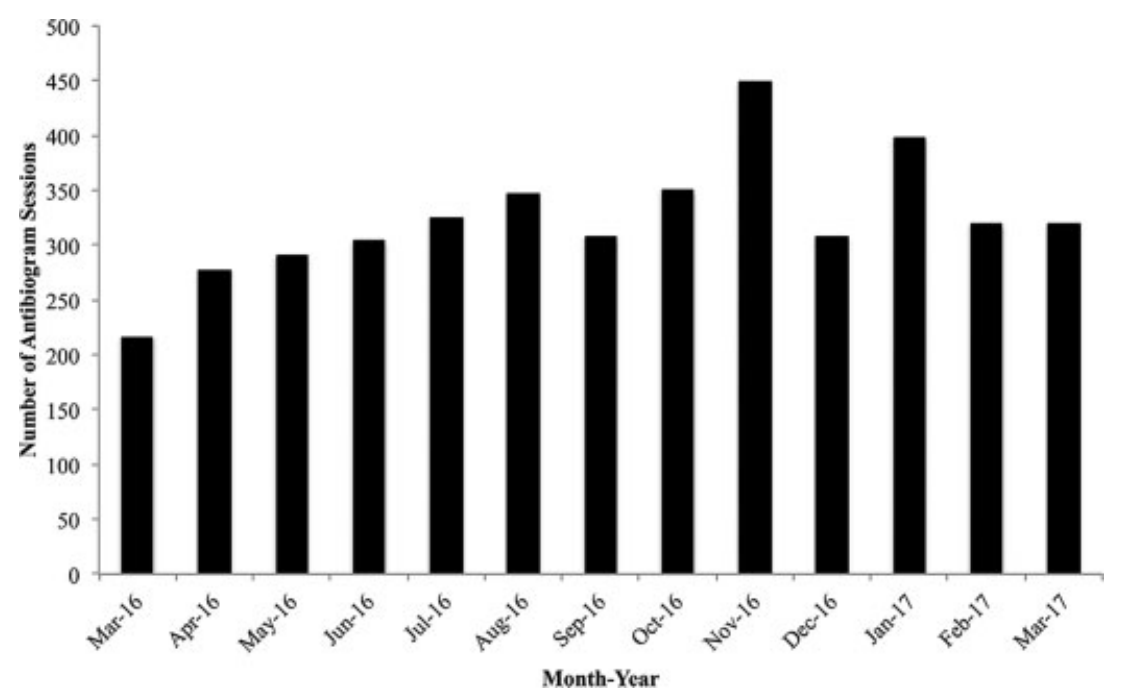

Fig. 4 Monthly electronic antibiogram aggregate session counts from March 2016 to March 2017.

Table 1 Categories of electronic antibiogram session users based on electronic health record login credentials from March 1, 2016 to March 31, 2017

\begin{tabular}{|l|l|l|}
\hline User category & Number of sessions & Percentage of total sessions \\
\hline Unknown-Workstation & 3,284 & 75 \\
\hline Physician house staff (fellows, residents, interns) & 415 & 9 \\
\hline Pharmacists, nurses & 331 & 8 \\
\hline Attending physicians & 244 & 6 \\
\hline Physician assistants and advanced practice nurses & 57 & 1 \\
\hline Medical and nursing students & 45 & 1 \\
\hline Total & 4,376 & 100 \\
\hline
\end{tabular}

Note: The "Unknown-Workstation" category refers to use of the electronic antibiogram at computer workstations where users' credentials cannot be tracked.

\section{Discussion}

We developed and implemented a novel, dynamic, e-antibiogram that has successfully and permanently taken the place of a yearly manual compilation of a static antibiogram. Our study has three main findings. First, it is feasible to implement a visual analytics e-antibiogram that supplies susceptibility maps for all organisms and antibiotics in a fully comprehensive report that is updated on a monthly basis, in contrast with a manually produced annual antibiogram that lists only the most common organisms and antibiotics. Second, an e-antibiogram can easily stratify laboratory data by source (wound, blood, or urine), hospital unit, and hospital- or community-acquired status, providing greater detail than a traditional antibiogram. Third, the e-antibiogram, which is accessed by a link in the EHR and thus available to all clinicians at all times, has logged an increasing number of sessions per month over a 12-month period.

Prompt, appropriate empirical antibiotic treatment improves patient outcomes, while the selection of unnecessary broad-spectrum antibiotics can increase harm to patients, antimicrobial resistance, and hospital costs. ${ }^{16}$ The marked escalation in the prevalence of antimicrobial resistance has made the selection of empirical antimicrobial therapy increasingly complex, and it is based large part on the susceptibility rates compiled in an institution's antibiogram. ${ }^{17}$ However, some manually compiled antibiograms might have outdated antibiotic susceptibility rates that can affect a clinician's selection of optimal empiric therapy. Our e-antibiogram may facilitate the selection of empiric therapy by delivering near real-time access to detailed information including organism speciation, antibiotic susceptibility, and the cost of antibiotic options.

We implemented filters for patient characteristics and unit locations as a novel solution to two potential shortcomings with a hospital-wide antibiogram. First, aggregate hospital data can mask differences in susceptibility data across specific patient characteristics-such as patient age and disease-and patient care units. Second, it can overlook pockets of resistance within a hospital if a highly resistant pathogen affects only one patient care unit. ${ }^{18,19}$ For example, pathogens that are isolated from patients with chronic 
diseases typically have higher rates of antimicrobial resistance than the pathogens obtained from patients without chronic diseases, and a similar pattern is often seen with pathogens from patients in intensive care units compared with patients in outpatient settings. The impact of this improved access to granular patient- and unit-specific data remains the focus of future study.

The e-antibiogram session data are encouraging and the use by nonprescribers suggests the tool's potentially broad utility. We anticipate that the rate of utilization will increase as we are actively promulgating the e-antibiogram as well as incorporating the e-antibiogram into clinical workflows such as embedding the e-antibiogram link in order sets. Unfortunately, analysis of the session data was hindered by the lack of identifiable user credentials at widely available "generic" clinical workstations. Preliminary workflow analysis has determined that attending physicians logged into the EHR more frequently from their hospital-owned computerswhich require an operating system login and thus contribute a greater proportion of identifiable antibiogram sessionsthan other health care providers who lack individually assigned hospital computers. This may also explain students' low percentage of identifiable sessions, as students are more likely to use the EHR at a generic clinical workstation that does not require an operating system login. Thus, the access patterns across the health care provider categories might differ significantly if the "unknown" logins were to be distributed accurately, and it is likely that users who typically lack assigned, hospital-owned computers (such as students, pharmacists, and nurses) constitute the majority of generic workstation e-antibiogram sessions.

Other available e-antibiograms include a Web-based antibiogram at Stanford University and a commercial offering called iAntibiogram (Teqqa, Jackson, Wyoming, United States). ${ }^{20,21}$ The Stanford e-antibiogram allows users to generate sensitivity tables from static yearly snapshots of data that can be filtered by organism and antimicrobial drug, but filtering by community versus hospital infections or on a rolling 12-month basis is unavailable, as our e-antibiogram offers. iAntibiogram delivers monthly updates as well as functionality such as filtering by hospital, unit, and source that are very similar to our e-antibiogram. Furthermore, iAntibiogram can be used on smartphones and the application can be installed locally, both functions that our e-antibiogram lacks.

It is important to note the limitations of both manually compiled and e-antibiograms to guide treatment in general. In many infections, such as pneumonia, the identification of the pathogen is the exception. Furthermore, sicker patients may be more likely to have a pathogen identified, which will bias any antibiogram toward more resistant isolates. Lastly, the best use of antibiogram data is not readily evident-for example, what percentage of resistant isolates in a given pathogen precludes empiric use of a certain agent? Unfortunately, fully addressing these points involves decision making beyond the implementation of an e-antibiogram. At CHOP, the selection of empiric therapies is done in multidisciplinary committees who collaborate to create clinical pathways. Antibiograms help to make data-driven decisions, but certainly other factors such as the severity of the patient's illness or immune status also play a role. Antibiograms can only relay data on isolates submitted to the microbiology laboratory. This is a limitation of both cumulative and specific antibiograms, yet they remain the only way to account for local patterns of resistance. However, despite their inherent limitations, antibiograms are excellent tools to help decision making once the organism is known. Prior to that, the user also has to take into account the likely frequency of the organisms that might grow in culture. Sitespecific data such as that presented in the e-antibiogram might help with this clinical challenge because the e-antibiogram can give relative frequencies of the different organisms at each site.

This study had several additional limitations. First, the eantibiogram was developed for use with our hospital's EHR data. Our results may not be fully generalizable to hospitals using other EHR systems. Second, while we have obtained the session data for the e-antibiogram, we have not assessed clinicians' empiric antibiotic prescribing patterns both before and after the implementation of the e-antibiogram. This will be the focus of future research. Third, while the traditional cut-off period for inpatients is less than 48 hours after hospital admission, we used a 72-hour cut-off period that is customary at our institution. Fourth, we did not track time efforts and costs associated with developing the e-antibiogram. Lastly, at this time the only user interaction that is tracked is the launching of the e-antibiogram within the EHR. The user's subsequent interactions with the e-antibiogram, such as selection of case scenarios and application of filters, cannot be tracked currently.

The e-antibiogram is an integral part of an ongoing quality improvement initiative at our hospital to optimize empiric antibiotic treatment based on our institution's EHR data. As users increasingly use the e-antibiogram, topics to be explored include antibiotic prescribing patterns, complications and errors associated with empiric antibiotic administration, and optimizing the e-antibiogram interface and design based on surveys, focus groups, and user feedback. Furthermore, there is a national effort among pediatric hospitals to share antimicrobial data and implement antimicrobial stewardship programs, and our e-antibiogram could be a useful tool to implement at other pediatric hospitals. $^{22,23}$ While we have shown that the development and implementation of an e-antibiogram can successfully replace the manual process of antibiogram compilation, our goal is to determine how the technology can best be utilized to improve clinical practice and ideally, patient outcomes.

\section{Conclusion}

An e-antibiogram that is generated and updated monthly from laboratory and pharmacy data are a feasible replacement for a yearly, static, manually compiled antibiogram. The e-antibiogram enables users to view stratified data in ways that can elucidate differences in susceptibility patterns that a traditional hospital-wide antibiogram cannot. The e-antibiogram has shown signs of increased usage over a 12 -month period. Future work will examine the impact of the e-antibiogram on antibiotic prescribing patterns. 


\section{Clinical Relevance Statement}

First, it is feasible to implement a visual analytics e-antibiogram that supplies susceptibility maps for all organisms and antibiotics in a fully comprehensive report that is updated on a monthly basis, in contrast with a manually produced annual antibiogram that lists only the most common organisms and antibiotics. Second, an e-antibiogram can easily stratify laboratory data by source (wound, blood, or urine), hospital unit, and hospital- or community-acquired status, providing greater detail than a traditional antibiogram. Third, stratifying data in an e-antibiogram can elucidate differences in susceptibility patterns that a traditional hospital-wide antibiogram cannot.

\section{Multiple Choice Question}

When constructing an interactive user interface that adheres to seminal visualization principles to achieve graphical excellence and integrity, it is crucial to do which of the following?

a. Vary spacing among and within cells that display data b. Use bright, playful colors for the areas framing data cells c. Select font types and sizes that maximize readability d. Use nonuniform cell sizes to maximize the use of space

Correct Answer: The correct answer is c. When constructing an interactive user interface that adheres to seminal visualization principles to achieve graphical excellence and integrity, it is crucial to eliminate distractors, determine the proper data density, and formulate an aesthetically pleasing "golden rectangle" interface design layout (Tufte ${ }^{12}$ ). The goal of these principles is to optimize the human-computer interaction by building an interface that is accessible, easy to use, and efficient.

Thus, font types and sizes in the interface should be selected carefully to maximize readability. Spacing among and within cells should remain uniform to avoid a haphazard arrangement of cells that might adversely affect users' processing of the tabular information. Cells containing data should maintain uniformity (if possible) for the same reason. Lastly, the areas framing data cells should be neutral so that the user's attention is not drawn away from the relevant information contained within the cells.

\section{Authors' Contributions}

All authors made significant contributions to the conception and design of the work. A.F.S., B.L., L.M.A., and J.A.G. made significant contributions to the acquisition of data used for analysis, performed data processing and data analysis, and interpretation of results. All authors contributed to drafting or revising the manuscript, approved the version to be published, and agree to be accountable for all aspects of the work.

\section{Protection of Human and Animal Subjects}

The study was performed in compliance with the World Medical Association Declaration of Helsinki on Ethical Principles for Medical Research involving human subjects, and was reviewed by the Children's Hospital of Philadelphia Institutional Review Board.

\section{Ethics Approval}

The Institutional Review Board at Children's Hospital of Philadelphia approved the study protocol.

\section{Funding}

This work was supported by departmental funding.

Conflict of Interest

None.

\section{References}

1 Hindler JF, Stelling J. Analysis and presentation of cumulative antibiograms: a new consensus guideline from the Clinical and Laboratory Standards Institute. Clin Infect Dis 2007;44(06):867-873

2 Kaye KS, Pogue JM. Infections caused by resistant Gram-negative bacteria: epidemiology and management. Pharmacotherapy 2015;35(10):949-962

3 National Action Plan for Combating Antibiotic-Resistant Bacteria. U.S. Centers for Disease Control. Available at: https://www.cdc. gov/drugresistance/pdf/national_action_plan_for_combating_antibotic-resistant_bacteria.pdf. Accessed August 2017

4 Jorgensen JH, Hindler JF. New consensus guidelines from the Clinical and Laboratory Standards Institute for antimicrobial susceptibility testing of infrequently isolated or fastidious bacteria. Clin Infect Dis 2007;44(02):280-286

5 CLSI. Analysis and Presentation of Cumulative Antimicrobial Susceptibility Test Data, Fourth Edition. CLSI document M39A4. Wayne, PA: Clinical and Laboratory Standards Institute; 2014

6 Tamma PD, Robinson GL, Gerber JS, et al. Pediatric antimicrobial susceptibility trends across the United States. Infect Control Hosp Epidemiol 2013;34(12):1244-1251

7 Binkley S, Fishman NO, LaRosa LA, et al. Comparison of unitspecific and hospital-wide antibiograms: potential implications for selection of empirical antimicrobial therapy. Infect Control Hosp Epidemiol 2006;27(07):682-687

8 Ahumada LM, Simpao AF, Galvez JA, et al. A visual analytics antibiogram dashboard as part of a comprehensive approach to perioperative antibiotic administration. Available at: https:// knowledge.amia.org/56638-amia-1.1540970/t-001-1.1545410/ f-001-1.1545411/a-001-1.1545676. Accessed September 6, 2017

9 Gálvez JA, Ahumada L, Simpao AF, et al. Visual analytical tool for evaluation of 10-year perioperative transfusion practice at a children's hospital.J Am Med Inform Assoc 2014;21(03):529-534

10 Simpao AF, Ahumada LM, Desai BR, et al. Optimization of drugdrug interaction alert rules in a pediatric hospital's electronic health record system using a visual analytics dashboard. J Am Med Inform Assoc 2015;22(02):361-369

11 Kimball R, Ross M, Thornthwaite W, Mundy J, Becker B, Eds. The Data Warehouse Lifecycle Toolkit. 2nd ed. Hoboken, NJ: Wiley; 2008

12 Tufte ER. The Visual Display of Quantitative Information. 2nd ed. Cheshire, CT: Graphics Press; 2001

13 Thomas JJ, Cook KA. A visual analytics agenda. IEEE Comput Graph Appl 2006;26(01):10-13

14 Wenzler E, Goff DA, Humphries R, Goldstein EJC. Anticipating the unpredictable: a review of antimicrobial stewardship and Acinetobacter infections. Infect Dis Ther 2017;6(02):149-172

15 Larru B, Ahumada LM, Metjian TA, et al. Comparison of a hospitalcumulative antibiogram with patient characteristic-stratified webbased antibiogram. Open Forum Infect Dis 2015;2(Suppl 1):82 
16 Cosgrove SE. The relationship between antimicrobial resistance and patient outcomes: mortality, length of hospital stay, and health care costs. Clin Infect Dis 2006;42(Suppl 2):S82-S89

17 Friedman ND, Temkin E, Carmeli Y. The negative impact of antibiotic resistance. Clin Microbiol Infect 2016;22(05):416-422

18 Swami SK, Banerjee R. Comparison of hospital-wide and age and location-stratified antibiograms of S. aureus, E. coli, and S. pneumoniae: age- and location-stratified antibiograms. Springerplus 2013;2(01):63

19 Kuster SP, Ruef C, Zbinden R, et al. Stratification of cumulative antibiograms in hospitals for hospital unit, specimen type, isolate sequence and duration of hospital stay. J Antimicrob Chemother 2008;62(06):1451-1461
20 Chen JH. Stanford Interactive Antibiogram. Available at: https:// web.stanford.edu/ jonc101/tools/Antibiogram/AntibiogramTable. htm. Accessed September 6, 2017

21 Teqqa. iAntibiogram. Available at: http://www.teqqa.com/iantibiogram/. Accessed September 6, 2017

22 Newland JG, Gerber JS, Kronman MP, et al; SHARPS Collaborative. Sharing Antimicrobial Reports for Pediatric Stewardship (SHARPS): a quality improvement collaborative. J Pediatric Infect Dis Soc 2017. Doi: 10.1093/jpids/pix020

23 Mistry RD, Newland JG, Gerber JS, et al. Current state of antimicrobial stewardship in children's hospital emergency departments. Infect Control Hosp Epidemiol 2017;38(04): 469-475 